\title{
Rezension: Beda Stähelin, Rechtsverfolgungs- kosten und unentgeltliche Rechtspflege im Lichte der Rechtsgleichheit
}

\author{
Matthias Schwaibold *
}

Der Autor untersucht die geltende Regelung über die unentgeltliche Rechtspflege unter dem Gesichtspunkt der Rechtsgleichheit. Seine Hauptthese, wonach die lex lata zu einer (neuen) Ungleichheit führe, weil sie den Mittelstand benachteilige, bleibt ohne empirische Belege. Die Vorschläge zur Behebung des unterstellten, nur theoretisch begründeten Mangels bleiben zaghaft, weshalb die Arbeit insgesamt nur einen geringen Beitrag zur aktuellen Diskussion über die prozessverhindernde Wirkung der Kostentragungsbestimmungen der ZPO leistet.

Zitiervorschlag: Matthias Schwaibold, Rezension: Beda Stähelin,

Rechtsverfolgungskosten und unentgeltliche Rechtspflege im Lichte der Rechtsgleichheit, in: sui-generis 2017, S. 260

URL: $\quad$ sui-generis.ch/48

DOI: $\quad$ https://doi.org/10.21257/sg.48

* Dr. Matthias Schwaibold, Rechtsanwalt Zürich. 
1 Ein Zivilprozess kostet Geld, und zunehmend viel Geld. Wer verliert, zahlt die Gerichtskosten, den eigenen Anwalt und den Anwalt der Gegenseite zumindest nach dem kantonalen Tarif, wer gewinnt, bleibt unter Umständen auf einem Teil der eigenen Anwaltskosten sitzen und verliert vielleicht sogar den von ihm geleisteten Prozesskostenvorschuss, weil das Gericht seine eigenen Kosten zuerst deckt und dabei nicht darauf schaut, ob Sieger oder Verlierer die Kostenvorschüsse geleistet haben. Aber nicht nur die grundsätzliche Kostenvorschusspflicht des Klägers und das Verlustrisiko bezüglich der von ihm geleisteten Kaution trotz Prozessgewinns werden zunehmend kritisiert. Auch die Höhe von Gerichtskosten und Parteientschädigungen haben in den letzten Jahren die (nicht gerade neue) Diskussion darüber belebt, wie weit die geltende Kostenregelung sich als «Prozessbremse», ja gar als Prozesshindernis erweist. Aus Kreisen der Richterschaft und der Zivilprozesslehre wird - weit deutlicher als von der Anwaltschaft - die Frage aufgeworfen, ob die bundeseinheitliche ZPO das Problem nicht noch verschärft hat und damit die Rechtsweggarantie in nicht mehr leichtem Masse aushöhlt oder bedroht.

Verbunden mit dieser ersten Frage ist eine zweite: Schaffen die Regeln über die unentgeltliche Prozessführung, die dem Mittellosen ermöglichen, einen nicht aussichtslosen Prozess zu führen, eine neue Ungleichheit? Wird nämlich derjenige, der zwar nicht mittellos ist, aber auch nicht gerade in Geld schwimmt, also der - prozesskostenmässig gesehen nicht Arme dadurch benachteiligt, weil der - prozesskostenmässig Arme - durch die unentgeltliche Prozessführung sozusagen zur geradezu reichen Gegenpartei wird, die frei von allen Kostenüberlegungen prozessieren kann, während der weder Arme noch Reiche sich über Gebühr durch die ihn treffenden Kostenfolgen in seiner prozessualen Bewegungsfreiheit eingeschränkt sieht?

3 Diese Fragen sind überaus berechtigt, aber auch nicht gerade von höchster theoretischer Schwierigkeit: Die Rechtsgleichheit gebietet nicht nur, dass dem Armen die Rechtsverfolgung zu ermöglichen ist, sie verbietet auch, dem nicht Armen die Prozessführung zu verunmöglichen. Oder anders herum: Die Rechtsgleichheit darf nicht dazu führen, dass nur noch die Armen dank der unentgeltlichen Rechtspflege und die Reichen dank sozusagen unbeschränkter Mittel Zivilprozesse sich leisten können, während ein (wie auch immer zu definierender) Mittelstand aus Kostengründen auf die Rechtsverfolgung faktisch verzichten muss.

4 In seiner Zürcher Dissertation spürt Beda Stähelin diesen Problemen nach: Das erste Kapitel (S. 1-9) stellt die Gliederung und Ziele der Arbeit vor, deren Ergebnisse im letzten, fünften Kapitel (S. 299-302) zusammengefasst werden. Im zweiten Kapitel (S. 11-54) wird die geltende Regelung über die Prozesskosten dargestellt, wobei naturgemäss die Regelungen über uP/uR im Vordergrund stehen. Das dritte Kapitel (S. 55-201) untersucht die geltende Regelung «im verfassungsrechtlichen Kontext», wobei der Autor insbesondere aufgrund von abstrakten Berechnungen die geltende Regelung als Prozessbremse erkennt (insb. zum Kostenrisiko S. 129 ff. und zur Kostenvorschusspflicht $\quad$ S. 132 ff.). Im vierten Kapitel (S. 203-297) wird die «unentgeltliche Rechtspflege im Lichte der Rechtsgleichheit» behandelt, was 
notwendigerweise zu - erneuten - Wiederholungen von Ausführungen aus den Kapiteln 2 und 3 führt.

Die Behauptung, dass «Geld eine (prozessuale) Waffe sei» (S. 188) und die nicht minder kühne These, wonach die geltende Ordnung des Zivilprozesses den wirtschaftlichen Stärkeren eine faktische Besserstellung bringe bzw. eine Chancenungleichheit zur Folge habe (S. 198), wird sowenig wie andere Thesen dieser Art wirklich begründet. Auch Antworten auf die eingangs dieser Besprechung gestellten Fragen bekommt man von Stähelin nicht. Er beschränkt sich darauf, solide den Stand der Diskussion darzustellen und in den Fussnoten vermutlich sehr vollständig die zugehörigen Nachweise aus Lehre und Rechtsprechung zu liefern. Einem eigenständigen Gedanken, der diese Diskussion in neue Bahnen lenken könnte, sucht man vergeblich - es sei denn, man wolle als diesen gelten lassen, dass Stähelin daran erinnert, dass gemäss Art. 118 Abs. 2 ZPO die unentgeltliche Rechtspflege ja auch nur teilweise gewährt werden könnte, es also keineswegs zwingend sei, sie entweder ganz oder gar nicht zu gewähren, wie es in der Praxis der Fall sei.

Und damit sind wir auch beim Kernproblem dieser Arbeit gelandet: Sie ist frei von allen rechtspraktischen, empirischen, rechtssoziologischen oder rechtstatsächlichen Befunden. Ein paar wenige reale oder fast reale Fallkonstellationen und die Einschätzungen, welche der Autor aus seiner eigenen Praxis und der von Kollegen hat, können diesen Mangel nicht wettmachen.

7 Hier wird ein zutiefst praktisches Problem auf einer gänzlich abstrakten, grund- rechtlichen Ebene abgehandelt, ohne dass man von diesem theoretischen Befund intellektuell besonders beeindruckt oder wenigstens vom Ergebnis überrascht sein könnte: Die Rechtsgleichheit verbietet es gleichermassen, Arme und Reiche ungleich zu behandeln, wie Reiche und Nichtreiche. Der geltenden Regelung über die unentgeltliche Prozessführung (allerdings relativ diskret) vorzuhalten, sie schaffe eine neue Ungleichheit, indem sie den «Mittelständler» sozusagen von der Rechtsverfolgung ausschliesst, lässt sich auf der theoretischen Ebene nicht beweisen. Natürlich ist es schwierig, gar nicht geführte Prozesse und die Motive, die zum Verzicht darauf führten, zu eruieren und zu quantifizieren; für prozessual und ausserprozessual geschlossene Vergleiche gilt dasselbe: Waren Kostenüberlegungen allein oder jedenfalls überwiegend massgeblich? Und es ist sicher auch nicht einfach, auf die Frage eine Antwort zu finden, ob ein Anwalt, der als Armenanwalt ohne Kostenrisiken prozessieren kann, ebenso prozessiert wie der Anwalt des von allen finanziellen Sorgen freien Klienten, während der Anwalt, der von seinem nicht über unbeschränkte Mittel verfügenden Klienten bezahlt wird, allein aus diesem Grund anders, sprich weniger kostenintensiv prozessiert.

8 Ebenso wäre doch zu prüfen, ob die Prozessführung des Armenanwalts immer derjenigen des «Reichenanwalts», um ein Unwort zu erfinden, entspricht, und ob sich die Prozessführung des vom «Mittelstandsanwalt» geführten Prozesses wirklich von derjenigen der vorgenannten unterscheidet. Lediglich diffuse Befürchtungen darüber, was sein und was nicht sein könnte, begründen weder 
einen gesetzgeberischen Handlungsbedarf noch belegen sie Dysfunktionalitäten.

9 Will man die geltende Regelung dafür verantwortlich machen, dass nicht begüterte Parteien auf die Durchsetzung ihrer Rechte verzichten und arme Parteien dazu ermuntert werden, frei von Kostenrisiken $\mathrm{zu}$ prozessieren, muss man das konkret aufzeigen können. Ansätze dazu könnten sein, dass man die Geschäftslast der Gerichte aus der Zeit vor 2011 mit der seit 2011 vergleicht, dass man untersucht, ob mehr Klagebewilligungen unbenützt verfallen als früher, dass man die bei den Gerichten hängigen Verfahren daraufhin untersucht, ob es relativ zur Gesamtheit der Verfahren mehr uP/uRFälle als früher gibt oder ob es Bereiche bzw. Streitwertgrössen gibt, die gegenüber früher signifikant weniger häufig geworden sind (wenn man so will: typische Mittelstandsfälle). Die Arbeit traktiert - vereinfacht - ein Rechtsgleichheitsproblem, ohne es empirischstatistisch zu beweisen.

Ebenfalls wenig Vergnügen bereitet die häufig umständliche und immer wieder geradezu hölzerne Sprache: «Das Erlangen eines besseren Verständnisses der prozessualen Erfolgsaussichten durch die Inanspruchnahme der vorsorglichen Beweisführung hat jedoch ihren Preis, denn für das nötige Verfahren und insbesondere die dabei getätigten Beweiserhebungen fallen selbstredend zu deckende Kosten an» (S. 116 Rz. 215).

11 Die wiederholt dargelegte Problematik, dass die unentgeltliche Rechtspflege denjenigen benachteilige, der gerade nicht mehr in ihren Genuss kommt, wäre dann eine, wenn sie tatsächlich bewiesen wäre. Sie wird nur argumentativ hergeleitet, aber das genügt nicht, die geltende Regelung als irgendwo mangelhaft auch zu belegen. Die vom Autor vorgeschlagene Abhilfe beschränkt sich im Wesentlichen darauf, einerseits einer tatsächlichen Beteiligung des vom Armenrecht Profitierenden an den Prozesskosten zu fordern, andererseits vermehrt eine lediglich teilweise Gewährung von $\mathrm{uP} / \mathrm{uR}$ zu postulieren.

12 Schliesslich wird mehr Grosszügigkeit bezüglich Ratenzahlung des kostenpflichtigen Klägers und bezüglich der Festlegung der «Armutsgrenze» gefordert, beides sozusagen zur Entlastung dessen, der nach der geltenden Regelung bzw. herrschenden Praxis (die auch in keiner Weise dargestellt wird!) gerade nicht mehr in den Genuss der uP/uR kommt. Der naheliegende Gedanke, dass die Rechtsgleichheit ja vielleicht dazu führen könnte, bei jeder Gewährung von uP/uR auch die Gegenpartei unter uP/uR prozessieren zu lassen, kam dem Rezensenten beim Lesen, nicht aber dem Autor. Damit soll nicht gesagt sein, dass das die Lösung aller (realen und unterstellten) Probleme wäre, aber das wäre dann zumindest ein Beitrag zur Diskussion.

13 Gesetzgeber, Gerichte und Öffentlichkeit kann man allerdings nur dann zum Handeln bewegen, wenn man neben rein theoretischen Argumenten auch belegte Tatsachen liefert. Jedenfalls letztere findet man bei Stähelin an keiner Stelle.

«Rechtsverfolgungskosten und unentgeltliche Rechtspflege im Lichte der Rechtsgleichheit dargestellt am Beispiel der Schweizerischen Zivilprozessordnung», Beat Stähelin, Diss. Zürich, Zürcher Studien zum öffentlichen Recht Bd. 250, Zürich, Schulthess 2017, ISBN: 978-3-7255-7705-7 